\title{
Group a Streptococcus Infection in Pregnancy
}

J uanillo EH ${ }^{1 *}$ and Chambliss LR $^{2}$

${ }^{1}$ Department of Obstetrics and Gynecology, Maricopa

Medical Center, Phoenix, Arizona, USA

${ }^{2}$ Department of Obstetrics and Gynecology, Division

of Maternal Fetal Medicine, St. J oseph's Hospital and

Medical Center, Arizona, USA

*Corresponding author: J uanillo EH, Department of Obstetrics and Gynecology, Maricopa Medical Center, Phoenix, Arizona, USA

Received: September 26, 2018; Accepted: October 26, 2018; Published: November 02, 2018

\begin{abstract}
Group A Streptococcus Infection (GAS) in pregnancy is a rare complication of pregnancy that is associated with significant morbidity and mortality in the peripartum period. Pregnant women have a 20 -fold increase in risk of severe GAS infection, and the course is rapid and may be fatal. In cases of GAS infection complicated by toxic shock syndrome, the mortality is estimated as high as $60 \%$. Early suspicion and intervention are key to improving outcomes in women with this invasive infection. Adoption of sepsis bundles in order to aid in early identification and prediction of patients that need intensive care is another cornerstone of management. This article aims to review the pathogenesis, diagnosis, and management of severe GAS infections in the peripartum period.
\end{abstract}

Keywords: Streptococcus Pyogenes; Pregnancy; Postpartum Sepsis; Toxic Shock Syndrome

\section{Introduction}

Although the morbidity and mortality of peripartum infection has decreased dramatically, infection with Group A Streptococcus (GAS) continues to have a significant contribution to peripartum complications. After initially decreasing with the advent of antiseptic technique and antibiotics, GAS has increased over the past few decades [1,2]. In the United Kingdom, GAS is the most common direct cause of maternal mortality [3]. Strategies for predicting and preventing infection have not been successful. Routine screening for GAS in selective populations has shown an incidence of $0.3-0.6 \%$ in pregnant women, which is significantly higher than the proportion of women who suffer from acute intrapartum infection [4]. Interestingly, this is much lower than the general population, who note an incidence of approximately $5-15 \%$. Conversely, the risk of invasive GAS infections is approximately 20 times higher in pregnant women than the general population [1]. The rates of GAS infection reported by the CDC from 2000-2004 was 3.5 cases per 100,000 persons [5]. The low incidence of severe GAS infection continues to make high-quality studies difficult, and the majority of the data surrounding peripartum GAS infections largely comes from case reports and epidemiologic studies.

\section{Pathogenesis}

Invasive GAS infections occur at an increased rate in pregnant women. The proposed mechanism of this is due to altered vaginal $\mathrm{pH}$ during pregnancy, damage to cutaneous barriers during labor and delivery as well as immuno suppressant during pregnancy [6]. Although risk factors such as prolonged rupture of membranes, diabetes mellitus, obesity have all been associated with severe infection and the increasing rates of obesity may contribute to variation in rates of GAS infection in developed countries.

Rarely present in vaginal micro biota, pathogenic GAS causes infection in the peripartum period it is associated with significant morbidity and mortality $[2,6]$. Virulence is attributed to the $M$ proteins, and emm1, emm28 are most commonly isolated in pregnant women [3]. M protein-fibrinogen complexes adhere to endothelium causing vascular leakages and hypercoagulability. In some cases, disseminated intravascular coagulopathy ensues, leading to more profound morbidity and mortality. In addition to this, $\mathrm{M}$ proteins allow the bacteria to avoid phagocytosis by macrophages and limit complement-mediated immune responses. GAS also produces exotoxin $\mathrm{A}$, which is responsible for toxic shock syndrome. Exotoxin A acts as a super antigen, which causes sudden release of inflammatory cytokines, capillary damage, and severe hypotension [7]. The increases in Prostaglandin E2 (PGE2) during has been implicated in the increased virulence of GAS during pregnancy. This protein is essential to maternal tolerance of foreign fetal tissues as well as cervical ripening and the onset of labor. Increased PGE2 inhibits macrophages, further decreasing phagocytosis of GAS bacteria and decreases local immune control of the disease $[8,9]$. Finally, increased maternal blood flow and increased oxygenation of the maternal genitourinary tract may also provide the media for GAS growth and pathogenic spread.

\section{Diagnosis and Management}

Outside of pregnancy, GAS infection presents as pharyngitis, rheumatic fever, and more rarely soft tissue infections and toxic shock syndrome [10]. During pregnancy, GAS infections most often present as endometritis, or rarely chorioamnionitis. Fever and uterine tenderness are the most common presenting symptoms. Invasive GAS infections progress rapidly and may be fatal within days of onset of symptoms. When a patient presents with symptoms of chorioamnionitis or endometritis that leads to rapid maternal deterioration, a high suspicion for GAS infection is imperative $[6,11]$. When culminating in Toxic shock syndrome, maternal cardiovascular collapse and multiorgan system failure follow the initial local infection. Laboratory studies in early infection are notable for leukocytosis $\left(>15,000 / \mathrm{mm}^{3}\right)$ and bandemia $(>10 \%)$. When GAS infection is complicated by Toxic Shock Syndrome (TSS) hypotension and multiorgan failure ensue $[1,6,11]$. Criteria for diagnosis of TSS includes hypotension, isolation of GAS from a normally sterile site and two or more signs of multiorgan involvement. Signs of multiorgan involvement include renal impairment (creatinine of $2 \mathrm{mg} /$ dLor doubling of baseline creatinine), coagulopathy (platelets 
of $100,000 / \mathrm{mm}^{3}$ or less, DIC), liver involvement (transaminitis or hyperbilirubinemia twice normal limits), ARDS, generalized erythematous macular rash that may or may not desquamate, and soft tissue necrosis $[6,10,11]$. The diagnosis of GAS strep infection is confirmed when the bacteria are isolated from maternal cultures. Most often, this is associated in blood cultures, but may also be noted in endometrial aspirates or amniotic fluid. In cases that initial present with pharyngitis, or pharyngeal swabs may first isolate the bacteria

Whenever GAS infection is suspected, prompt treatment with antibiotics is indicated. Streptococcus continues to be sensitive to penicillin, and treatment with an infusion of 4 million units every four hours is usually adequate. Since penicillin targets the production of cell wall proteins in bacteria, it targets bacteria that are growing and replicating [10-12]. This leads to the "eagle effect," which describes decreased efficacy for bacteria in the stationary phase of their life cycle. The addition of clindamycin to the antibiotic regimen is suggested for serious peripartum infections $[10,11]$. There is limited data to support the addition of IVIG in severe GAS infections, and there is conflicting evidence that this improves mortality $[10,11]$. In conjunction with prompt antibiotic administration, source control is an integral part of management of severe GAS infections. In the puerperium, the most common source is the uterus. There is limited evidence comparing conservative management and hysterectomy for GAS infection, and hysterectomy is often necessary for adequate source control [11]. The mortality of TSS in the first 48 hours postpartum is as high as $60 \%$, and hysterectomy has the potential of significantly decreasing the bacterial inoculums and improving response to antibiotic therapy $[13,14]$. Deheane et al suggest that serial monitoring of creatinine kinase as a measure of myometrial involvement. As the patient clinically deteriorated, CK increased and the decision was made to proceed with hysterectomy. After surgical intervention, CK normalized in the patient and the authors conclude that this may be a reliable marker to help guide management in similar cases [15]. Otherwise, the decision to proceed with surgical management is dictated by clinical course and rapid response to conservative management. In cases of softtissue infection of intraabdominal abscesses, source control should be attempted with debridement or drainage as applicable [16].

As national attention turns to maternal mortality and systembased interventions to address the most common causes of maternal death, the application of sepsis bundles to maternity care is increasingly recommended. Initially published in 2004, the Surviving Sepsis Campaign describes time-sensitive bundles aimed at decreasing mortality from sepsis and septic shock [17,18]. Over a decade of evidence supports application of these interventions in the emergency care and inpatient management. Expert opinion in obstetrics and gynecology supports application of these bundles to management of pregnant patients. The 2018 update of these guidelines recommends an "Hour-1 bundle," which recommends measuring lactate, obtaining blood cultures, antibiotic administration, rapid fluid resuscitation, and vasopressors when mean arterial pressure is less than $65 \mathrm{mmHg}$. [17] After initial management, source control should be attempted, and assembly of a multidisciplinary team is essential to guiding further management. Critical care specialists and anesthesia providers are indispensable members of a multidisciplinary approach to invasive GAS infection and the management of TSS $[10,11,17]$. The use of venous-arterial extracorporeal membrane oxygenation in postpartum GAS infection has been described and may be useful in unstable patients that do not respond to conventional management and vasopressors [18].

\section{Future Directions}

With the exception of evolving sepsis guidelines, little changes to the diagnosis and management of severe peripartum GAS infections has been made in the last two decades. Increasing attention is focused on preventative strategies. Prevention strategies that rely on early screening tools are most effective for diseases that are common in the general population. The low prevalence of vaginal colonization and the low incidence of infection has made this a challenging avenue of study. At this point GAS screening is not recommended, although studies show that it is feasible in conjunction with Group B strep screening [19]. Alternatively, the development of a GAS vaccine is ongoing [20]. Current vaccine trials target pharyngitis in the pediatric population and rheumatic fever. Similar to the advent of the MMR vaccine and congenital rubella infections, it is not unreasonable to consider that introduction of the GAS vaccine to the public at large may decrease the incidence of severe peripartum GAS infections.

\section{Conclusion}

Pregnant women have a 20 -fold higher risk of severe GAS infections due maternal physiology and disruption of maternal barriers to infection. These infections continue to have a significant contribution to maternal morbidity and mortality. High suspicion of GAS infection in the peripartum period is essential to rapid intervention and prevention of severe complications. In addition, implementation of sepsis bundles in the management of pregnant patients aims to decrease the complications associated with these complications. Early notification and consultation of critical care providers in cases of severe sepsis and TSS is essential to address the sequelae of this infection and prevent morbidity and mortality. Until preventative strategies beyond aseptic technique are identified, early identification and rapid application of standardized sepsis management is essential in preventing catastrophic maternal outcomes.

\section{References}

1. Shinar S, Fouks Y, Amit S, Pauzner D, Tarabeia J, Schechner V, et al. Clinical Characteristics of and Preventative Strategies for Peripartum Group A Streptococcal Infections: Obstetrics \& Gynecology. 2016; 127: 227-232.

2. Sosa MEB. Group A Streptococcal Infection in Pregnancy and the Puerperium: The Journal of Perinatal \& Neonatal Nursing. 2016; 30: 124-130.

3. Turner CE, Dryden M, Holden MTG, Davies FJ, Lawrenson RA, Farzaneh L, et al. Molecular Analysis of an Outbreak of Lethal Postpartum Sepsis Caused by Streptococcus pyogenes. Journal of Clinical Microbiology. 2013; 51: 20892095.

4. Deutscher M, Lewis M, Zell ER, Taylor TH, Van Beneden C, Schrag S, et al. Incidence and Severity of Invasive Streptococcus pneumoniae, Group A Streptococcus, and Group B Streptococcus Infections Among Pregnant and Postpartum Women. Clinical Infectious Diseases. 2011; 53: 114-123.

5. O'Loughlin RE, Roberson A, Cieslak PR, Lynfield R, Gershman K, Craig A, et al. The Epidemiology of Invasive Group A Streptococcal Infection and Potential Vaccine Implications: United States, 2000-2004. Clinical Infectious Diseases. 2007; 45: 853-862.

6. Anderson BL. Puerperal Group A Streptococcal Infection: Beyond Semmelweis. Obstetrics \& Gynecology. 2014; 123: 874-882. 
7. Golińska E, van der Linden M, Więcek G, Mikołajczyk D, Machul A, Same $A$, et al. Virulence factors of Streptococcus pyogenes strains from women in peri-labor with invasive infections. European Journal of Clinical Microbiology \& Infectious Diseases. 2016; 35: 747-754.

8. Mason KL, Aronoff DM. Postpartum Group A Streptococcus Sepsis and Maternal Immunology: Postpartum Group A Streptococcus Sepsis. American Journal of Reproductive Immunology. 2012; 67: 91-00.

9. Mason KL, Rogers LM, Soares EM, Bani-Hashemi T, Erb Downward J, Agnew D, et al. Intrauterine Group A Streptococcal Infections Are Exacerbated by Prostaglandin E2. The Journal of Immunology. 2013; 191: 2457-2465.

10. Steer AC, Lamagni T, Curtis N, Carapetis JR. Invasive Group A Streptococca Disease: Epidemiology, Pathogenesis and Management. Drugs. 2012; 72 1213-1227.

11. Hamilton SM, Stevens DL, Bryant AE. Pregnancy-Related Group A Streptococcal Infections: Temporal Relationships Between Bacterial Acquisition, Infection Onset, Clinical Findings, and Outcome. Clinical Infectious Diseases. 2013; 57: 870-876.

12. Levy MM, Evans LE, Rhodes A. The Surviving Sepsis Campaign Bundle: 2018 Update. Critical Care Medicine. 2018; 46: 997-1000.

13. Hasegawa J, Sekizawa A, Yoshimatsu J, Murakoshi T, Osato K, Ikeda T, et al. Cases of death due to serious group A streptococcal toxic shock syndrome in pregnant females in Japan. Archives of Gynecology and Obstetrics. 2015; 291: 5-7.

14. Rimawi BH, Soper DE, Eschenbach DA. Group A Streptococcal Infections in Obstetrics and Gynecology: Clinical Obstetrics and Gynecology. 2012; 55: 864-874.
15. Dehaene I, Loccufier A, Temmerman M, De Keersmaecker B, De Baene L. Creatine Kinase as an Indicator for Hysterectomy in Postpartum Endomyometritis due to Group A Streptococci: A Hypothesis Illustrated by a Case Report. Gynecologic and Obstetric Investigation. 2012; 73: 82-88.

16. Acosta CD, Knight M. Sepsis and maternal mortality: Current Opinion in Obstetrics and Gynecology. 2013; 25: 109-116

17. Dellinger RP, Carlet JM, Masur H, Gerlach H, Calandra T, Cohen J, et al Surviving Sepsis Campaign guidelines for management of severe sepsis and septic shock. Intensive care medicine. 2004; 30: 536-555.

18. Imaeda T, Nakada T, Abe R, Tateishi Y, Oda S. Veno-arterial extracorporeal membrane oxygenation for Streptococcus pyogenes toxic shock syndrome in pregnancy. Journal of Artificial Organs. 2016; 19: 200- 233

19. Saab J, Bell SM, Lahra MM. Vaginal carriage rate of streptococcal pyogenes in 1600 pregnant women. Pathology. 2012; 44: 567-568.

20. Dale JB, Batzloff MR, Cleary PP, Courtney HS, Good MF, Grandi G, et al Current Approaches to Group A Streptococcal Vaccine Development. In: Ferretti JJ, Stevens DL, Fischetti VA, editors. Streptococcus pyogenes : Basic Biology to Clinical Manifestations [Internet]. Oklahoma City (OK): University of Oklahoma Health Sciences Center; 2016.
Austin J Obstet Gynecol - Volume 5 Issue 8 - 2018

Submit your Manuscript | www.austinpublishing group.com

Juanillo et al. (C) All rights are reserved
Citation: Juanillo EH and Chambliss LR. Group a Streptococcus Infection in Pregnancy. Austin J Obstet Gynecol. 2018; 5(8): 1126 